\title{
Decreased activation of inflammatory networks during acute asthma exacerbations is associated with chronic airflow obstruction
}

\author{
A Bosco $^{1,2}$, S Ehteshami ${ }^{1,2}$, DA Stern ${ }^{1,2}$ and FD Martinez ${ }^{1,2}$
}

Asthma exacerbations are associated with subsequent deficits in lung function. Here, we tested the hypothesis that a specific pattern of inflammatory responses during acute exacerbations may be associated with chronic airway obstruction. Gene coexpression networks were characterized in induced sputum obtained during an acute exacerbation, from asthmatic children with or without chronic airflow limitation. The data showed that activation of Th1-like/cytotoxic and interferon signaling pathways during acute exacerbations was decreased in asthmatic children with deficits in baseline lung function. These associations were independent of the identification of picornaviruses in nasal secretions or the use of medications at the time of the exacerbation. Th2-related pathways were also detected in the responses, but variations in these pathways were not related to chronic airways obstruction. Our findings show that decreased activation of Th1-like/cytotoxic and interferon pathways is a hallmark of acute exacerbation responses in asthmatic children with evidence of chronic airways obstruction.

\section{INTRODUCTION}

In most cases of asthma, the first symptoms of the disease occur during childhood, ${ }^{1}$ and in a subgroup of children, the disease is associated with deficits in airway function that track with age and predict persistence of asthma symptoms into adult life. ${ }^{2-4}$ There is now strong evidence suggesting that these deficits are acquired during the course of the disease, ${ }^{5}$ are more likely to become apparent during childhood, ${ }^{6}$ and that chronic administration of inhaled corticosteroids does not prevent them. ${ }^{7}$ Moreover, at least one-third of older asthmatics develop chronic obstructive pulmonary disease, ${ }^{8}$ and among that third, airflow limitation is often present in early adult life. ${ }^{9}$ Thus, a better understanding of the molecular mechanisms that may be associated with airflow limitation in children with asthma could help design new treatments to prevent persistent asthma and asthmaassociated chronic obstructive pulmonary disease.

Recent longitudinal studies have suggested that, among children and adults with asthma, those with a higher incidence of acute exacerbations are more likely to have subsequent deficits of lung function growth ${ }^{10}$ or excess decline, ${ }^{11}$ respectively. The mechanisms that underlie the associations between exacerbations and chronic lung disease in humans are unknown.
Evidence of infection with rhinoviruses has been reported in $50-70 \%$ of asthma exacerbations, ${ }^{12}$ and accumulating data suggest that immune responses to viruses may be altered in asthmatics. When infected with rhinovirus, bronchial epithelial cells obtained from adult atopic asthmatics have deficits in interferon type I and III responses. ${ }^{13,14}$ Impaired interferon- $\gamma$ (IFN- $\gamma$ ) and augmented Th2 responses in blood and BAL T cells from asthmatics are associated with rhinovirus-induced clinical illness severity and viral load. ${ }^{15}$ Rhinovirus-induced IFN- $\gamma /$ IL-5 response ratios in peripheral blood mononuclear cell cultures in vitro are positively correlated with forced expiratory volume in $1 \mathrm{~s}$ (FEV1), ${ }^{16}$ and these same response ratios in sputum are inversely related to viral clearance in vivo. ${ }^{17} \mathrm{~A}$ recent study in mice showed that respiratory viral infections can trigger the development of a chronic asthma/chronic obstructive pulmonary disease-like lung disease after the virus is cleared to trace levels. ${ }^{18}$ In this mouse model, invariant natural killer T (iNKT) cells have a central role in disease pathogenesis; nevertheless, there are conflicting data on the role of iNKT cells in the pathogenesis of human asthma. ${ }^{19,20}$

We postulated that children whose asthma is associated with chronic airflow limitation could have a specific pattern of

\footnotetext{
${ }^{1}$ Arizona Respiratory Center, College of Medicine, University of Arizona, Tucson, Arizona, USA. ${ }^{2}$ BIO5 Institute, University of Arizona, Tucson, Arizona, USA. Correspondence: A Bosco (abosco@arc.arizona.edu) 
inflammatory responses in the airways that predisposes them to the development of deficits in airway function. ${ }^{21}$ To test this hypothesis, we induced sputum at the time of a moderate exacerbation and 7-14 days later in a group of children with asthma followed prospectively, and compared global patterns of gene expression in sputum cells in those with or without baseline airflow limitation. Our results show that the activation of Th1like/cytotoxic and IFN signaling pathways during exacerbations is decreased in asthmatic children with evidence of chronic airflow obstruction. Moreover, regardless of the presence or absence of chronic airflow obstruction, acute exacerbations were associated with markers of iNKT cells, and the latter were highly correlated with cytokines that promote Th1/cytotoxic responses (IL-12A, IL-21).

\section{RESULTS}

\section{Study population and follow-up}

The study population consisted of $218 \mathrm{mild} /$ moderate persistent asthmatic children aged 6-18 years who had at least one acute asthma exacerbation during the previous year. Of these 218 children, 117 experienced an exacerbation during the observation period, and sputum samples from 40 of these subjects were available for this study. Of all baseline characteristics, only ethnicity, maternal smoking, and frequency of ever having been hospitalized for asthma were different between included and excluded children who had exacerbations (Table 1).

At enrollment, modest correlations were observed between FEV1/forced vital capacity (FVC) ratio and height and age $(\rho=-0.33, P$ value $=0.035$ and $\rho=-0.37, P$ value $=0.020$, respectively). There was no statistically significant relation between ethnicity, gender, parental asthma, current smoking, skin test reactivity, detection of picornavirus in nasal secretions, ever hospitalized for asthma, or concurrent medication use and the baseline FEV1/FVC ratio (Supplementary Table S1 online).

\section{Gene coexpression network analysis of exacerbation responses: variations associated with FEV1/FVC ratio}

Two groups of 10 subjects each from both extreme tails of the FEV1/FVC distribution at baseline were selected (see Methods section) and their mean \pm s.d. FEV1/FVC ratios were 78.8 \pm 2.1 and $91.3 \pm 2.5$, respectively. Total RNA was extracted from induced sputum samples obtained from these subjects during an exacerbation, and the samples were labeled and hybridized to microarrays. A holistic approach was used to analyze the microarray data based on reverse engineering gene network analysis. $^{22-24}$ The mechanics of the network analysis algorithm are detailed in Methods section; briefly, the algorithm uses a stepwise analytical process to interrogate variations in gene coexpression patterns across all the samples to identify subnets of coexpressed genes or modules. These modules contain the molecular components that execute biological functions and underpin physiological and diseased states. ${ }^{22-24}$ As shown in Figure 1a, network analysis of exacerbation responses in sputum identified 12 modules of coexpressed genes. To determine if any of the modules were differentially expressed in responses from
Table 1 Characteristics of the participants who had an exacerbation and were included and not included in the present study

\begin{tabular}{|c|c|c|c|}
\hline Characteristics & $\begin{array}{l}\text { Included, \% } \\
(n+/ n \text { total) }\end{array}$ & $\begin{array}{l}\text { Not included, } \\
\%(n+/ n \text { total })\end{array}$ & $\begin{array}{c}P \\
\text { value }\end{array}$ \\
\hline \multicolumn{4}{|l|}{ Enrollment } \\
\hline \multicolumn{4}{|l|}{ Ethnicity, \% } \\
\hline Hispanic white & $70.0(28 / 40)$ & $77.9(60 / 77)$ & 0.02 \\
\hline Non-Hispanic white & $7.5(3 / 40)$ & $11.7(9 / 77)$ & \\
\hline African American & $2.5(1 / 40)$ & $7.8(6 / 77)$ & \\
\hline Alaska native & $15.0(6 / 40)$ & $2.6(2 / 77)$ & \\
\hline$>1$ race & $5.0(2 / 40)$ & $0.0(0 / 77)$ & \\
\hline \multicolumn{4}{|l|}{ Gender, \% } \\
\hline Female & $45.0(18 / 40)$ & $44.2(34 / 77)$ & 0.3 \\
\hline Male & $55.0(22 / 40)$ & $55.8(43 / 77)$ & \\
\hline $\begin{array}{l}\text { Age, years, mean } \pm \text { s.d. } \\
(n)\end{array}$ & $\begin{array}{l}11.0 \pm 3.4 \\
(40)\end{array}$ & $10.1 \pm 2.7(77)$ & 0.1 \\
\hline $\begin{array}{l}\text { Height, cm, mean } \pm \text { s.d. } \\
(n)\end{array}$ & $\begin{array}{c}143.5 \pm 17.5 \\
(40)\end{array}$ & $\begin{array}{c}139.4 \pm 17.8 \\
(74)\end{array}$ & 0.2 \\
\hline $\begin{array}{l}\text { Aeroallergen skin test } \\
\text { positive, \% }\end{array}$ & $73.0(27 / 37)$ & $80.3(57 / 71)$ & 0.5 \\
\hline FEV1/FVC ratio, \% (n) & $\begin{array}{c}84.8 \pm 5.7 \\
(40)\end{array}$ & $83.1 \pm 6.8(75)$ & 0.2 \\
\hline Ever eczema, \% & $42.5(17 / 40)$ & $53.9(41 / 76)$ & 0.3 \\
\hline Ever Hay fever, \% & $45.0(18 / 40)$ & $52.6(40 / 76)$ & 0.6 \\
\hline $\begin{array}{l}\text { Ever hospitalized for } \\
\text { asthma, \% }\end{array}$ & $15.0(6 / 40)$ & $36.0(27 / 75)$ & 0.02 \\
\hline \multicolumn{4}{|l|}{ Medications, \% } \\
\hline $\begin{array}{l}\text { Inhaled corti- } \\
\text { costeroids }\end{array}$ & $30.0(12 / 40)$ & $36.4(28 / 77)$ & 0.5 \\
\hline LTRA & $20.0(8 / 40)$ & $26.0(20 / 77)$ & 0.5 \\
\hline Advair & $32.5(13 / 40)$ & $28.6(22 / 77)$ & 0.7 \\
\hline \multicolumn{4}{|l|}{ Parental ever asthma, \% } \\
\hline Maternal & $27.5(11 / 40)$ & $32.9(25 / 76)$ & 0.7 \\
\hline Paternal & $15.0(6 / 40)$ & $15.8(12 / 76)$ & 0.9 \\
\hline \multicolumn{4}{|l|}{$\begin{array}{l}\text { Parental current } \\
\text { smoking, \% }\end{array}$} \\
\hline Maternal & $5.0(2 / 40)$ & $22.4(17 / 76)$ & 0.02 \\
\hline Paternal & $35.0(14 / 40)$ & $36.8(28 / 76)$ & 0.9 \\
\hline \multicolumn{4}{|l|}{ Exacerbation } \\
\hline $\begin{array}{l}\text { Time to exacerbation, } \\
\text { days, mean } \pm \text { s.d. }(n)\end{array}$ & $\begin{array}{l}114 \pm 124 \\
(40)\end{array}$ & $\begin{array}{l}134.5 \pm 118 \\
(77)\end{array}$ & 0.4 \\
\hline $\begin{array}{l}\text { Picornavirus positive, } \\
\%(n)\end{array}$ & $47.2(17 / 36)$ & $43.1(22 / 51)$ & 0.8 \\
\hline FEV1/FVC ratio, \% ( $n)$ & $\begin{array}{l}83.8 \pm 5.9 \\
\quad(36)\end{array}$ & $81.4 \pm 8.8(63)$ & 0.2 \\
\hline \multicolumn{4}{|l|}{ Medications, \% } \\
\hline $\begin{array}{l}\text { Inhaled corti- } \\
\text { costeroids }\end{array}$ & $35.0(14 / 40)$ & $31.2(24 / 77)$ & 0.7 \\
\hline $\begin{array}{l}\text { Leukotriene recep- } \\
\text { tor antagonist }\end{array}$ & $17.5(7 / 40)$ & $20.8(16 / 77)$ & 0.8 \\
\hline $\begin{array}{l}\text { Combination } \\
\text { therapy }\end{array}$ & $37.5(15 / 40)$ & $24.7(19 / 77)$ & 0.2 \\
\hline
\end{tabular}

Table 1 continued on the following page 
Table 1 (Continued)

\begin{tabular}{|c|c|c|c|}
\hline Characteristics & $\begin{array}{l}\text { Included, \% } \\
(n+/ n \text { total) }\end{array}$ & $\begin{array}{l}\text { Not included, } \\
\%(n+/ n \text { total })\end{array}$ & $\begin{array}{c}P \\
\text { value }\end{array}$ \\
\hline \multicolumn{4}{|l|}{ Convalescent } \\
\hline $\begin{array}{l}\text { Exacerbation to } \\
\text { convalescent, days, } \\
\text { mean } \pm \text { s.d. }(n)\end{array}$ & $\begin{array}{c}13.6 \pm 3.8 \\
(40)\end{array}$ & $15.6 \pm 7.5(68)$ & 0.1 \\
\hline FEV1/FVC ratio, \% (n) & $\begin{array}{l}84.8 \pm 6.0 \\
(36)\end{array}$ & $82.4 \pm 6.0(64)$ & 0.1 \\
\hline \multicolumn{4}{|l|}{ Medications, \% } \\
\hline $\begin{array}{l}\text { Inhaled corti- } \\
\text { costeroids }\end{array}$ & $42.5(17 / 40)$ & $42.6(29 / 68)$ & 0.9 \\
\hline $\begin{array}{l}\text { Leukotriene recep- } \\
\text { tor antagonist }\end{array}$ & $17.5(7 / 40)$ & $22.1(15 / 68)$ & 0.6 \\
\hline $\begin{array}{l}\text { Combination } \\
\text { therapy }\end{array}$ & $35.0(14 / 40)$ & $33.8(23 / 68)$ & 0.9 \\
\hline
\end{tabular}

Abbreviations: FEV1, forced expiratory volume in $1 \mathrm{~s}$; FVC, forced vital capacity.

subjects with or without deficits in FEV1/FVC ratios, we tested the modules with Gene Set Analysis ${ }^{25}$ - a statistical procedure that tests for the association of a set of genes with a trait of interest. This analysis provided preliminary evidence that three modules were associated with enrollment FEV1/FVC ratios (modules II, III, and IV, Figure 1b), and accordingly these modules were selected for further study.

The first module (module III, Figure 1b) was decreased in subjects with low FEV1/FVC ratios as compared with those with normal FEV1/FVC ratios at baseline, and it was designated the "Th1-like/cytotoxic pathway" because it was enriched for signatures associated with Th1/IFN- $\gamma$ (CCL8, CCR5, CXCL9, CXCL10, indoleamine 2,3-dioxygenase, IFN- $\gamma$, IL-12RB2, SLAM, SOCS1, STAT4, TxK) ${ }^{26}$ and cytotoxic responses (killer cell lectin-like receptor-1, IL-15, IL-21, granulysin, granzyme $\mathrm{B}$, granzyme $\mathrm{K}$, perforin-1). The second module (module IV, Figure 1b), "IFN signaling pathway", was also decreased in subjects with low FEV1/FVC ratios as compared with those with normal FEV1/FVC ratios at baseline, and it was mainly composed of genes downstream of IFN signaling (IFN-induced proteins (35, 44, 44L, H1, I6, T1, T3, T5, TM3), IRF-7, Mx1, OAS2, OAS3, OASL, protein kinase R (EIF2AK2), PML, STAT2, TRAIL). The third module (module II, Figure 1b) was elevated in subjects with low FEV1/FVC ratios as compared with those with normal FEV1/FVC at baseline, and it was designated the "epithelial differentiation pathway" because it was enriched for genes that are associated with the mucociliary differentiation of airway epithelial cells (ECM1, ELF3, CLCA4, DUOX2, FAM3D, HSPB8, KRT3, KRT4, KRT16, MAL, MUC1, PPL, RCHG, SCEL, SERPINB2, SPRR1A, SPRR3). ${ }^{27}$ The Th1-like/cytotoxic, IFN signaling, and epithelial differentiation pathways contained 115,78 , and 71 genes respectively, and the complete set of genes in these pathways are listed in Supplementary Tables S2-S4 online. The stability of the three selected modules was tested using a leave-one-out procedure as detailed in Methods section and was greater than $80 \%$ for the "Th1-like/cytotoxic" and "epithelial differentiation" modules. The stability of the "IFN
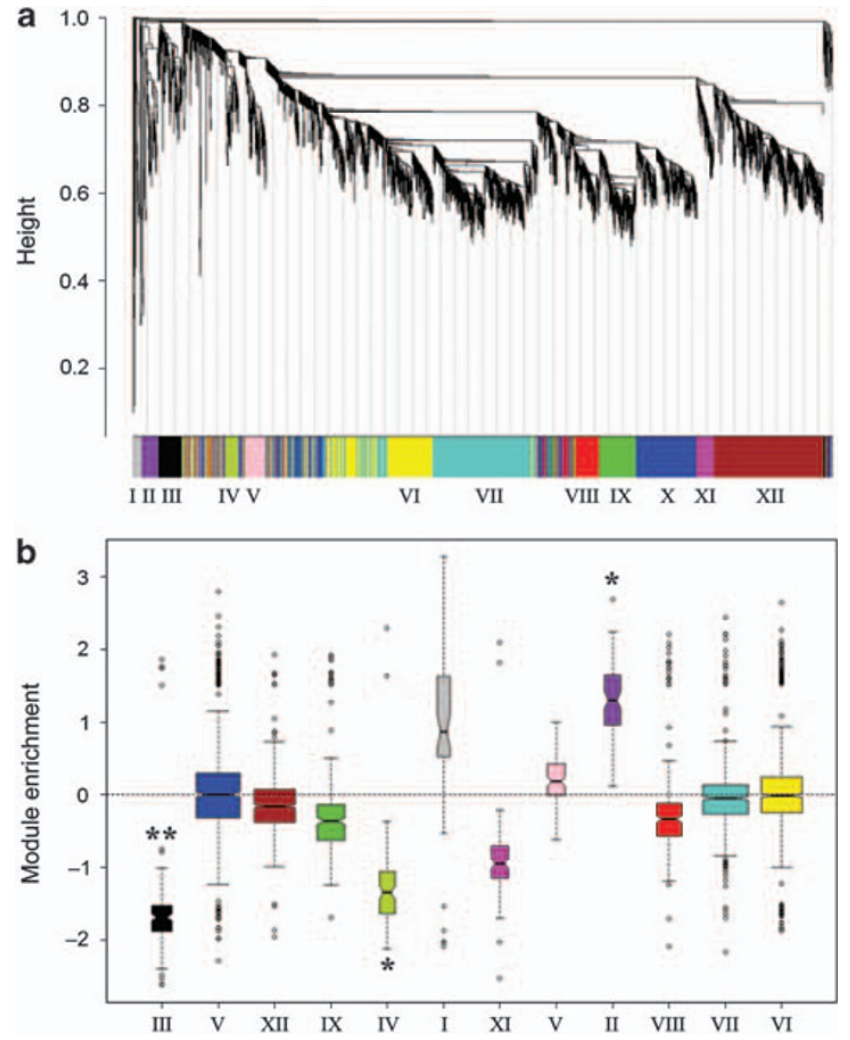

Figure 1 Exacerbation responses are associated with baseline forced expiratory volume in $1 \mathrm{~s} /$ forced vital capacity (FEV1/FVC) ratios. Gene expression was profiled by microarray in sputum samples obtained during an acute exacerbation from asthmatic children with $(n=10)$ or without $(n=10)$ deficits in enrollment/baseline FEV1/FVC ratios. (a) Modules of coexpressed genes were identified by reverse engineering gene network analysis of the microarray data set $(n=20)$. (b) The modules were tested for differential expression in responses from subjects with or without deficits in baseline FEV1/FVC ratios. Positive or negative values on the vertical axis indicate that a module is enriched with genes that are increased or decreased, respectively (based on gene-level Bayesian $t$-test statistics ${ }^{46}$ ) in responses from subjects with deficits in FEV1/FVC ratios as compared with those with normal FEV1/FVC ratios. The $P$ values are derived from a statistical analysis at the module-level using the Gene Set Analysis test without adjustment for multiple testing. ${ }^{29 * *} P$ value $<0.01 ;{ }^{*} P$ value $<0.05$.

signaling" module was lower at 63\%; however, this cluster contained a highly conserved core comprising an archetypal IFNinduced gene signature (see Supplementary Table S3 online).

Th2-related pathways were not detected in the network analyses; however, a conventional differential expression analysis of the microarray data suggested that FCER1A and IL-5 were elevated in the responses from subjects with low FEV1/FVC ratios (Supplementary Figure S1 online). Therefore, we also selected Th2-related genes (FCER1A, IL-5, $I L-13)$ for further study.

\section{Gene expression patterns during acute exacerbations and at 7-14 days convalescence}

The microarray analyses above were based on sputum samples obtained during an acute exacerbation, thus it is not known if the gene expression signals are transient/activation-associated, 
Table 2 Expression levels of representative genes from the Th1-like/cytotoxic, interferon signaling, and Th2related pathways are upregulated in sputum during acute exacerbations in comparison to 7-14 days convalescence

\begin{tabular}{|c|c|}
\hline Gene symbol & Biological function \\
\hline \multicolumn{2}{|c|}{ Th1-like/cytotoxic pathway } \\
\hline $\begin{array}{l}\text { CXCL10 } \\
(I P-10)\end{array}$ & $\begin{array}{l}\text { Highly expressed during rhino- } \\
\text { virus-induced asthma exacer- } \\
\text { bations. Attracts CD4T, CD8T, } \\
\text { and NKT cells via CXCR3 }\end{array}$ \\
\hline IFN- $\beta$ & $\begin{array}{l}\text { IFN- } \alpha / \beta \text { induce a robust anti- } \\
\text { viral state, are essential for } \\
\text { immunity to most viruses, and } \\
\text { are potent inducers of cytotoxic } \\
\text { responses }\end{array}$ \\
\hline$I F N-\gamma$ & $\begin{array}{l}\text { Principal Th1 cytokine and } \\
\text { macrophage-activating factor. } \\
\text { Essential for immunity to myco- } \\
\text { bacterium and some viruses }\end{array}$ \\
\hline$I L-2 R \alpha$ & $\begin{array}{l}\text { IL-2 signaling regulates } \\
\text { immune responses through } \\
\text { the maintenance of Treg and } \\
\text { induction of activation-induced } \\
\text { cell death, promotes the pro- } \\
\text { liferation and differentiation of } \\
\text { NK and T cells, and programs } \\
\text { antiviral CD8T cell memory } \\
\text { responses }\end{array}$ \\
\hline$I L-15$ & $\begin{array}{l}\text { Role in development, growth, } \\
\text { homeostasis, and cytotoxic } \\
\text { function of CD8T cells, NK and } \\
\text { iNKT cells }\end{array}$ \\
\hline IL-21 & $\begin{array}{l}\text { Enhances cytotoxic function of } \\
\text { CD8T cells, NK and } \mathbb{N} K T \text { cells. } \\
\text { Essential for control of chronic } \\
\text { viral infections. Regulates IgE } \\
\text { responses }\end{array}$ \\
\hline $\begin{array}{l}\text { GZMB } \\
\text { (granzyme B) }\end{array}$ & $\begin{array}{l}\text { Principal mediator of CD8T cell } \\
\text { and NK cytotoxic responses. } \\
\text { Role in antiviral defense }\end{array}$ \\
\hline $\begin{array}{l}\text { PRF1 } \\
\text { (perforin-1) }\end{array}$ & $\begin{array}{l}\text { Delivers cytotoxic granules } \\
\text { containing granzyme B to } \\
\text { induce apoptosis of target } \\
\text { cells. Essential for immunity to } \\
\text { some viruses }\end{array}$ \\
\hline
\end{tabular}

Exa vs Conv $P$ value $^{a}$

0.0004

0.0182

0.0013

0.0009

0.0006

0.0024

0.0011

0.0090

Interferon signaling pathway

Mx1 Induced by IFN- $\alpha,-\beta,-\lambda$. Traps viral components and inhibits viral replication

PKR

(EIF2AK2)

Induced by IFN- $\alpha,-\beta,-\lambda$. Inhibits protein translation through phosphorylation of EIF2 $\alpha$

PML Induced by IFN- $\alpha / \beta$ and IFN- $\gamma$, forms nuclear bodies and inhibits viral replication

CCL2 Induced by IFN- $\alpha / \beta$. Essential

(MCP-1) for recruitment of macrophages and protection of the alveolar epithelium during respiratory viral infections
Table 2 Continued

\begin{tabular}{|c|c|c|}
\hline Gene symbol & Biological function & $\begin{array}{c}\text { Exa vs Conv } \\
P \text { value }\end{array}$ \\
\hline \multicolumn{3}{|c|}{ Epithelial differentiation pathway } \\
\hline ECM1 & $\begin{array}{l}\text { Loss-of-function mutations in } \\
\text { ECM1 cause Urbach-Wiethe } \\
\text { disease, a rare, autosomal } \\
\text { recessive disorder character- } \\
\text { ized by abnormalities in skin } \\
\text { and mucous membranes }\end{array}$ & 0.8875 \\
\hline KRT4 & $\begin{array}{l}\text { Structural protein expressed in } \\
\text { differentiated layers of mucosal } \\
\text { and esophageal epithelia }\end{array}$ & 0.8192 \\
\hline SPRR3 & Marker of squamous epithelium & 0.6243 \\
\hline TMPRSS11D & $\begin{array}{l}\text { Trypsin-like protease } \\
\text { expressed in bronchial epithe- } \\
\text { lial cells. May have a role in } \\
\text { airway remodeling }\end{array}$ & 0.3550 \\
\hline \multicolumn{3}{|c|}{ Th2-related pathways } \\
\hline FCER1A & $\begin{array}{l}\text { High-affinity receptor subunit } \\
\text { for IgE, which is upregulated } \\
\text { on Mo/DC during viral-driven } \\
\text { exacerbations in children and } \\
\text { on DC in viral-driven lung dis- } \\
\text { ease in mice }\end{array}$ & 0.0060 \\
\hline IL-5 & $\begin{array}{l}\text { Role in the pathogenesis of } \\
\text { exacerbations in eosinophilic, } \\
\text { refractory form of asthma }\end{array}$ & 0.0084 \\
\hline$I L-13$ & $\begin{array}{l}\text { Mediates cardinal features of } \\
\text { asthma and viral-driven lung } \\
\text { diseases in mice. Role in the } \\
\text { pathogenesis of allergen- } \\
\text { driven late phase response in } \\
\text { humans }\end{array}$ & 0.0512 \\
\hline
\end{tabular}

aNormalized gene expression levels as measured by qRT-PCR were compared in paired sputum samples obtained from asthmatic children $(n=18)$ during an exacerbation (Exa) or 7-14 days convalescence (Conv). Statistical analyses by Wilcoxon's signed-rank test. Undetected data points were substituted for half the lowest value. See Supplementary Table S5 online for references.

or constitutive. To obtain more detailed information in this regard, we profiled expression of representative genes with known immunological functions from the identified pathways, by real-time quantitative reverse transcription PCR (qRT-PCR) in paired acute and convalescent samples, which were available for a subset of the study population $(n=18)$. The responses for all 18 subjects combined are shown in Table 2 (see Figure 2 for illustrative examples), and these data showed that representative genes in the Th1-like/cytotoxic pathway, the IFN signaling pathway, and the Th2 pathway (FCER1A, $I L-5)$ were significantly elevated in acute vs. convalescent comparisons, and there was also a trend for increased levels of IL-13 $(P$ value $=0.051)$. In contrast, genes in the epithelial differentiation pathway were not significantly modulated in acute vs. convalescent comparisons (Table 2). Of note, expression of the housekeeping gene $H M B S$ that was used to normalize the qRT-PCR data to adjust for variations in RNA quantity and quality was not different in acute vs. convalescent comparisons $(P$ value $=0.92)$. 

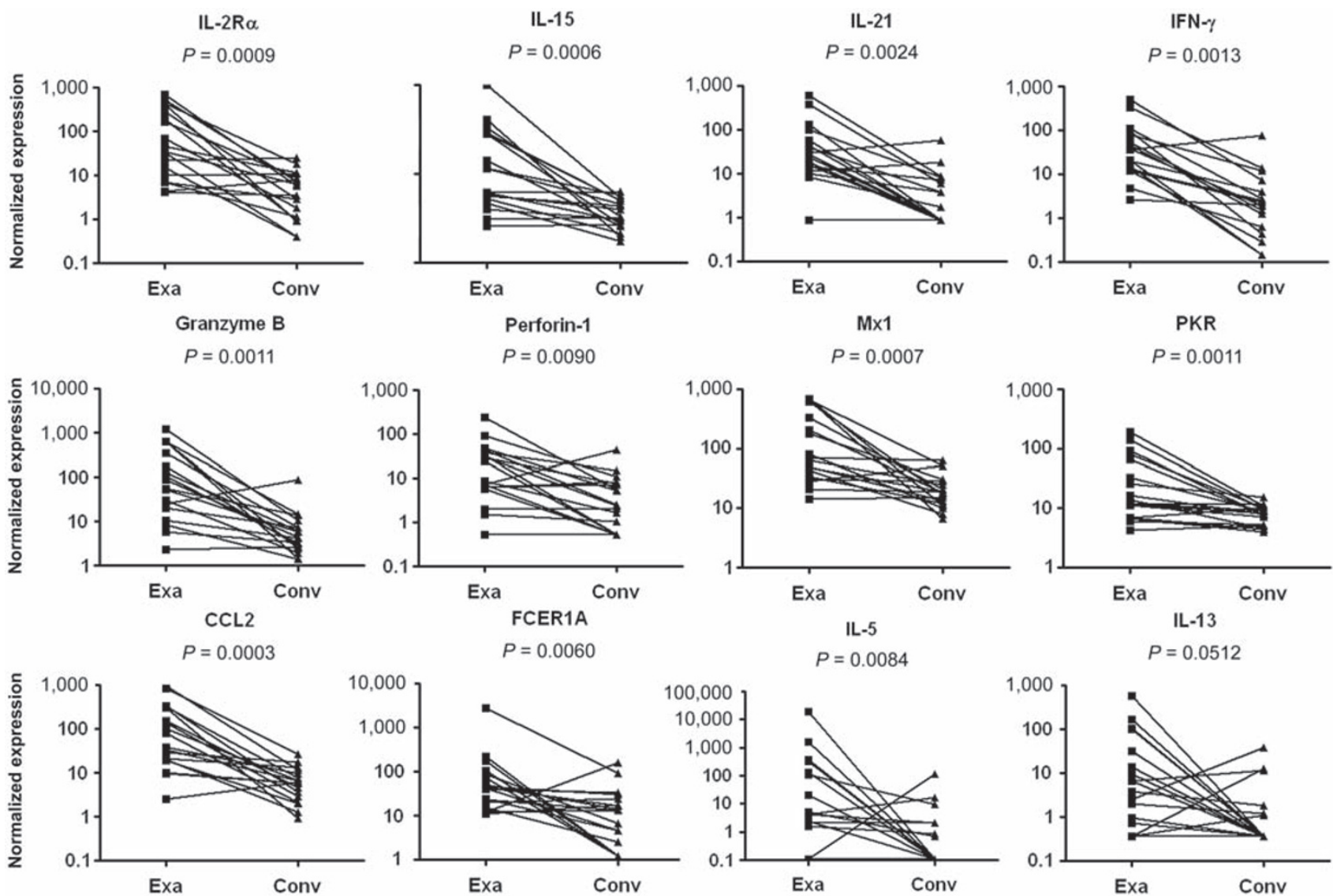

Figure 2 Inflammatory genes are upregulated in sputum during acute exacerbations of asthma in comparison to 7-14 days convalescence. Normalized gene expression levels were measured by qRT-PCR in paired sputum samples obtained from asthmatic children ( $n=18)$ during an acute exacerbation (Exa) and 7-14 days later (Conv). Statistical analysis by Wilcoxon's signed-rank test. Undetected data points were substituted for half the lowest value.

Stratification of the subjects (from Table 2/Figure 2) into FEV1/FVC tertiles showed that the responses for representative genes from the Th1-like/cytotoxic and IFN signaling pathways were much more consistent and/or intense in subjects with higher baseline FEV1/FVC ratios (Supplementary Figures S2-S6 online). Moreover, statistical analyses detected consistent associations between FEV1/FVC ratios at enrollment, and exacerbation responses for genes from the Th1-like/cytotoxic and IFN signaling pathways, but not from the epithelial differentiation or Th2-related pathways (Supplementary Figure S7 online).

\section{qRT-PCR analysis of exacerbation responses and variations in FEV1/FVC ratios}

We next sought confirmation of the preliminary associations between exacerbation response patterns and enrollment FEV1/FVC ratios detected by microarray in Figure 1b, using real-time qRT-PCR analysis of all available samples from the whole population. Of note, these subjects exhibit a broad range of FEV1/FVC ratios, thus in the analyses below FEV1/FVC was treated as a quantitative trait. The nonparametric analyses showed in Table 3 confirmed that expression of representative genes from the Th1-like/cytotoxic and IFN signaling pathways during exacerbations was strongly and positively correlated with enrollment FEV1/FVC ratios. However, we could not show that similar correlations were observed with FEV1/FVC ratios at the time of the exacerbations, when the sputum samples themselves were obtained. There were no associations between expression of genes from the epithelial differentiation and Th2-related pathways with FEV1/FVC ratios assessed at any stage. No association was detected with FEV1/FVC ratios and expression of the housekeeping gene HMBS.

Inclusion in the analyses of sputum samples that contain a higher proportion of contaminating squamous cells may decrease power to detect true associations, and this could potentially explain the lack of association between the epithelial and/or Th2-related pathways with FEV1/FVC ratios. To address this issue, we excluded samples that contained more than $30 \%$ squamous cells, repeated the analyses, and the results were unchanged (Supplementary Table S6 online).

Variations in gene expression could potentially be explained by variations in the clinical characteristics of the study population. However, no consistent associations were found between gene expression levels and skin test reactivity, or evidence of concurrent picornavirus infection, or use of specific medications at the time of exacerbation (Supplementary Table S7 online). 
Table 3 Expression levels of Th1-like/cytotoxic and interferon signaling genes in sputum during acute exacerbations are correlated with enrollment and convalescent FEV1/FVC ratios, but not with exacerbation FEV1/FVC ratios

\begin{tabular}{|c|c|c|c|c|c|c|}
\hline \multirow[b]{2}{*}{ Gene symbol } & \multicolumn{6}{|c|}{ FEV1/FVC ratio } \\
\hline & $\rho$ & $P$ value $^{a}$ & $\rho$ & $P$ value ${ }^{a}$ & $\rho$ & $P$ value ${ }^{a}$ \\
\hline CXCL10 (IP-10) & 0.44 & 0.004 & 0.10 & 0.6 & 0.48 & 0.003 \\
\hline IFN- $\beta 1$ & 0.38 & 0.016 & 0.25 & 0.1 & 0.41 & 0.01 \\
\hline IL-15 & 0.45 & 0.003 & 0.04 & 0.8 & 0.53 & 0.001 \\
\hline$I L-21$ & 0.52 & 0.001 & 0.18 & 0.3 & 0.58 & $<0.001$ \\
\hline GZMB (granzyme B) & 0.50 & 0.001 & 0.05 & 0.8 & 0.54 & 0.001 \\
\hline PRF1 (perforin-1) & 0.53 & $<0.001$ & 0.22 & 0.2 & 0.52 & 0.001 \\
\hline$P M L$ & 0.41 & 0.009 & 0.01 & 0.9 & 0.49 & 0.002 \\
\hline CCL2(MCP-1) & 0.45 & 0.004 & 0.10 & 0.6 & 0.52 & 0.001 \\
\hline \multicolumn{7}{|c|}{ Epithelial differentiation pathway } \\
\hline ECM1 & -0.03 & 0.9 & 0.04 & 0.8 & -0.03 & 0.9 \\
\hline KRT4 & -0.15 & 0.4 & -0.11 & 0.5 & -0.17 & 0.3 \\
\hline SPPR3 & 0.05 & 0.8 & 0.10 & 0.6 & -0.06 & 0.7 \\
\hline TMPRS11D & -0.03 & 0.8 & 0.04 & 0.8 & -0.03 & 0.9 \\
\hline \multicolumn{7}{|l|}{ Th2-related pathways } \\
\hline FCER1A & -0.12 & 0.5 & -0.18 & 0.3 & 0.15 & 0.4 \\
\hline
\end{tabular}

${ }^{a}$ Gene expression levels were measured by qRT-PCR in sputum samples obtained from asthmatic children during an exacerbation. Statistical analysis by Spearman's rank correlation, unadjusted. Undetected data points were substituted for half the lowest value. High-quality lung function data were available for all subjects at enrollment, but were either missing or did not meet ATS guidelines for some subjects during the exacerbation or at convalescence.

\section{Exacerbation responses, specific medications, and FEV1/ FVC ratios}

Linear regression modeling was used to investigate associations between exacerbation response patterns and FEV1/FVC ratios, while adjusting for medications used at the time of the exacerbation. Consistent with the unadjusted analyses in Table 3, we identified strong and positive associations between the Th1like/cytotoxic and IFN pathways and enrollment/convalescent FEV1/FVC ratios, and again, no associations were detected between FEV1/FVC ratios and either Th2 or epithelial differentiation pathways (Supplementary Table S8 online).

\section{Cellular immune signatures enriched within the Th1-like/ cytotoxic pathway}

To attempt to infer the cellular origins of the gene expression signals in the Th1-like/cytotoxic pathway, we used a bioinformatics "in silico" approach using a publicly available database (see Methods section). Previous studies have documented high expression levels of the cytotoxic pathway in CD4T, CD8T, and NK cells from blood, ${ }^{28}$ and in these same cell populations isolated from the airways during respiratory viral infections. ${ }^{29}$ Our bioinformatics analyses (Supplementary Table $\mathbf{S 9}$ online) showed that the list of genes in the Th1-like/cytotoxic pathway was significantly enriched for molecular signatures associated with antiviral responses $\left(P\right.$ value $\left.=1.13 \times 10^{-9}\right), \mathrm{CD} 4 \mathrm{~T}$ cells $\left(P\right.$ value $\left.=1.05 \times 10^{-9}\right)$, cytotoxic CD8T cells $\left(P\right.$ value $\left.=1.63 \times 10^{-5}\right)$, T-cell receptor signaling $\left(P\right.$ value $\left.=2.7 \times 10^{-4}\right)$, and NK-cellmediated cytotoxicity $\left(P\right.$ value $\left.=1.77 \times 10^{-5}\right)$. However, we also identified a signature for iNKT cells $\left(P\right.$ value $\left.=1.04 \times 10^{-5}\right)$ in the Th1-like/cytotoxic pathway. In addition, two genes (SLAM, $S A P / S H 2 D 1 A$, Supplementary Table $\mathbf{S} 2$ online) that are central to the development of iNKT cells ${ }^{30}$ are also part of the Th1like/cytoxic network.

To obtain more detailed information on the role of $\mathrm{T}$ cells and iNKT cells in exacerbation responses, we investigated expression of T-cell receptor transcripts by qRT-PCR in the 

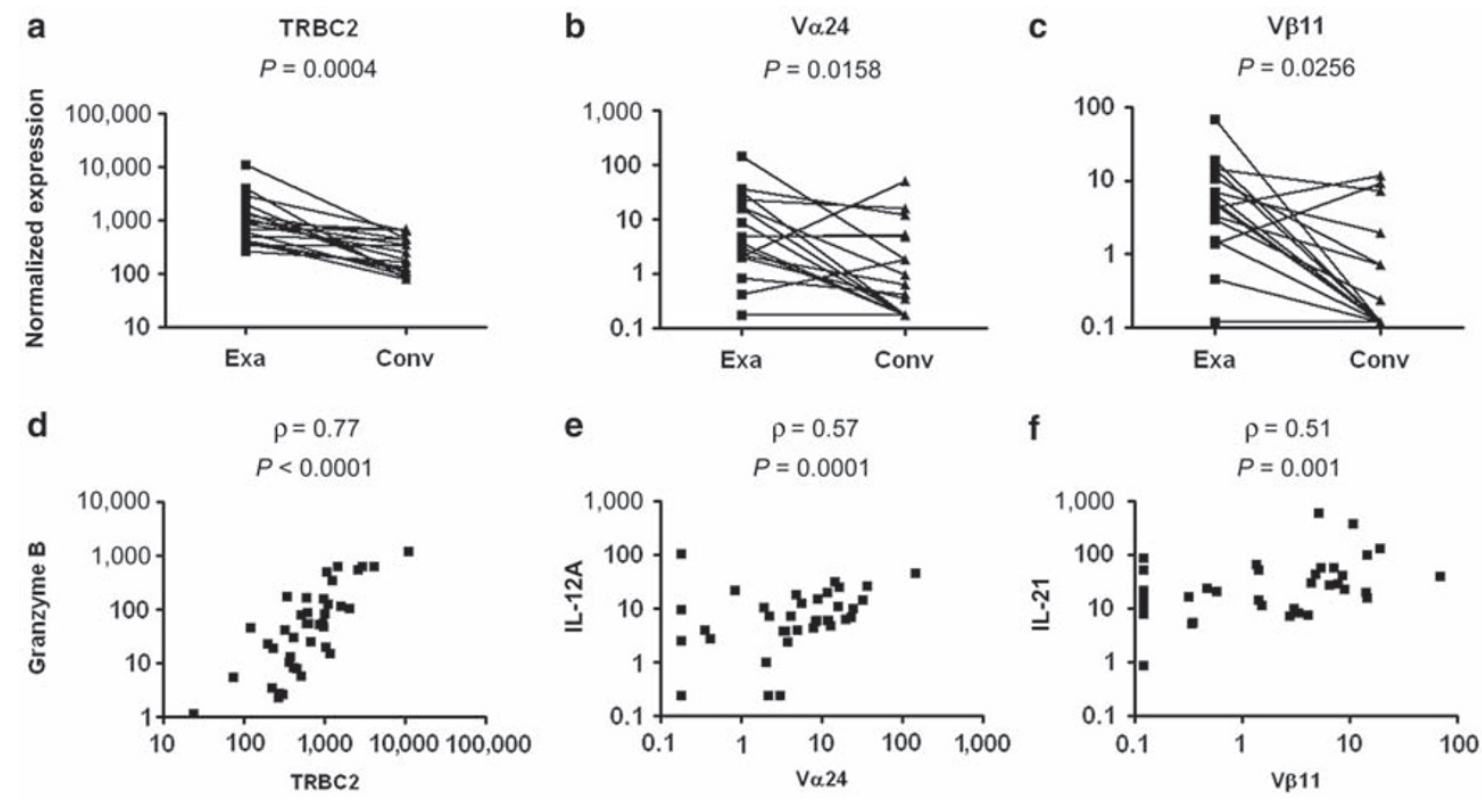

Figure 3 Markers of T cells and invariant natural killer T (iNKT) cells are upregulated during exacerbations; the latter are highly correlated with IL-12A and IL-21 responses. (a-c) Normalized gene expression levels measured by qRT-PCR in paired sputum samples obtained from asthmatic children ( $n=18$ ) during an acute exacerbation (Exa) and at 7-14 days convalescence (Conv). Statistical analysis by Wilcoxon's signed-rank test. (d-e) Normalized gene expression levels measured by qRT-PCR in sputum obtained during an acute exacerbation from asthmatic children ( $n=40)$. Statistical analysis by Spearman's rank correlation. Undetected data points were substituted for half the lowest value.

sputum samples. ${ }^{20}$ Expression of the T-cell receptor constant chain (TRBC2) - a marker of T cells including iNKT cellswas detected in all exacerbation and convalescent samples, and expression levels were significantly elevated during exacerbations (Figure 3a). Expression of the iNKT cell markers $\mathrm{V} \alpha 24$ and V $\beta 11$ was detected in 80 and $70 \%$ of the exacerbation responses, respectively (data not shown), and once again expression levels were significantly elevated during exacerbations (Figure $\mathbf{3 b}$ and $\mathbf{c}$ ).

Finally, we investigated associations between expression of T-cell and iNKT cell markers, exacerbation response patterns, and FEV1/FVC ratios. These analyses showed that TRBC2 and iNKT cell markers were not related to FEV1/FVC ratios (data not shown); however, the former was strongly and positively correlated with the activation of Th1-like/cytotoxic and IFN pathways, and the latter were strongly correlated with IL-12A and IL-21 responses (Supplementary Table S10 online; see Figure $3 \mathbf{e}$ and $\mathbf{f}$ for illustrative examples).

\section{DISCUSSION}

Childhood asthma is a heterogeneous condition characterized by recurrent episodes of reversible airway obstruction. In a substantive proportion of children with asthma, the disease is associated with progressive airflow limitation, as assessed by the development of deficits in FEV1/FVC ratio, a useful index of the remodeling state of the airway unrelated to anthropometric data. ${ }^{31}$ What determines this irreversible loss of lung function is unknown, but a recent longitudinal study in children and adults with asthma of recent onset suggested that those who had exacerbations during follow-up were at increased risk. ${ }^{10}$ The purpose of this study was thus to determine if children with baseline airflow obstruction had a pattern of inflammatory responses during acute asthma exacerbations that was different from that of children without baseline airway obstruction. We showed that decreased activation of Th1-like/cytotoxic and IFN signaling pathways during acute asthma exacerbations were strongly associated with chronic airways obstruction. These associations were independent of atopy, the detection of concurrent picornavirus infections, and the use of medications at the onset of the exacerbation. Th2-related pathways were also detected in the exacerbation responses (Figure 2; Supplementary Figure S1 online), but variations in these pathways were not related to FEV1/FVC ratios. Interestingly and unexpectedly, the patterns of association with lung function observed between acute gene expression and FEV1/FVC ratios at baseline and during convalescence were not observed with FEV1/FVC ratio measured during the acute episode. We speculate that determinants of the severity of the acute obstructive response may be different from those that influence long-term effects on lung function. Finally, bioinformatics analyses of cellular immune signatures enriched within the Th1-like/cytotoxic pathway suggested a role for T cells and iNKT cells in the exacerbation responses, and we showed that the activation of Th1-like/cytotoxic and IFN signaling networks was strongly correlated with a marker of T cells (TRBC2). Markers of iNKT cells (V $\alpha 24, \mathrm{~V} \beta 11$ ) were also associated with acute exacerbations; although they were not related to airways obstruction, they were strongly correlated with IL-12A and IL-21 responses. These results thus suggest that impairment of acute Th1-like/cytotoxic and IFN signaling responses presumably to viruses and other environmental exposures may have a major role in the development of chronic airways obstruction in asthma. 
Our findings confirm and extend previous studies showing deficient rhinovirus-induced IFN responses in asthmatic adults in vivo. ${ }^{14,15,17}$ Of particular interest were the studies by Wark et al. ${ }^{13}$ who compared IFN- $\beta$ responses, induction of apoptosis, and viral release after rhinovirus infection in bronchial epithelial cells obtained from asthmatic adults with and without airflow limitation (with only the former requiring treatment with inhaled corticosteroids) and normal controls. They found that, whereas IFN- $\beta$ and apoptotic responses were impaired in both groups with asthma, viral release from these cells was significantly increased only among asthmatic subjects with airflow limitation. Contoli et al. ${ }^{14}$ reported deficits in IFN- $\lambda$ responses and increased viral shedding by rhinovirus-infected bronchial epithelial cells obtained from subjects with asthma who had mild baseline airflow limitation. These results thus suggested that deficits in several innate inflammatory responses might facilitate virus replication and cytolysis, with increased infection of neighboring airway cells, thus inducing exaggerated secondary responses, which in turn may activate remodeling and abnormal repair mechanisms. Our study addresses this potential complexity by adopting an unbiased approach to the assessment of inflammatory networks that are set in motion during real-life asthma exacerbations in children. We show that the Th1-like and IFN pathways function in the broader molecular context of the cytotoxic pathway (granulysin, granzyme B, perforin-1) and common $\gamma$ chain cytokine signaling pathways (IL-2Ra, IL-15, IL-21), which are known to enhance the differentiation, proliferation, and/or cytotoxic functions of CD4T, CD8T, NK, and iNKT cells (Table 2). It is also noteworthy that IFN- $\beta$ in addition to inducing a robust antiviral state by upregulation of the archetypal IFN-induced genes $(M x 1, P K R, P M L)$ also potently activates cytotoxic responses. ${ }^{32}$ Thus, activation of a complex network of interrelated immune mechanisms seems to be necessary to protect subjects with asthma from the development of airflow limitation after acute asthma exacerbations.

The detection of iNKT cells in sputum during exacerbations is not all that surprising, given their established role in immune responses against respiratory infections ${ }^{18,33}$ and to a broad range of other pathogens. ${ }^{34}$ Our findings show that markers of iNKT cells are strongly associated with IL-12A and IL-21 responses (Supplementary Table S10 online; Figure 3e and f). Activated iNKT cells potently induce production of the Th1-promoting cytokine IL- 12 by dendritic cells, ${ }^{35}$ and IL- 12 drives iNKT celldependent cytotoxic responses. ${ }^{36}$ IL-21 is highly expressed by CD4T cells and iNKT cells, ${ }^{37}$ and IL-21 enhances the cytotoxic functionality of NK and iNKT cells. ${ }^{37,38}$ In the direct context of viral infections, deficits in IL-21 responses in cord blood are associated with increased risk for severe respiratory infections. ${ }^{39}$ Moreover, studies in animal models have shown that the IL-21 pathway is essential to sustain antiviral responses and control persistent infections. ${ }^{40} \mathrm{On}$ the basis of the strong association of iNKT cell markers with IL-12A and IL-21 responses, we hypothesize that iNKT cells have a major role in coordinating protective Th1-like and cytotoxic responses during exacerbations.

Studies in animal models have suggested a pathogenic role for iNKT cells in asthma. ${ }^{18,41}$ However, immune responses in mice do not always mimic those in humans, ${ }^{42,43}$ and data on iNKT cells in human asthma are limited and conflicting. ${ }^{19,20}$ iNKT cells were significantly increased during exacerbations in our data, but this association was modest due to evidence for the persistence of these cells in sputum 7-14 days after the exacerbation in a subset of the subjects (Figure $\mathbf{3 b}$ and $\mathbf{c}$ ). This was in contrast to a broader marker for $\mathrm{T}$ cells, which was reduced to relatively low levels at 7-14 days convalescence (Figure 3a). Unfortunately, numbers of subjects and duration of follow-up were insufficient to determine if persistence of markers for the presence of iNKT cells was associated with chronic airflow limitation, as suggested by mouse models. Further studies are needed to examine the relationship between acute asthma exacerbations, chronic airway obstruction, and numbers of iNKT cells and $\mathrm{T}$ cells in the airways.

This study has limitations that should be acknowledged. In our study, the abnormalities in lung function were already present at enrollment, and thus, a cause-effect relation between the inflammatory patterns we observed and these abnormalities cannot be proven using our study design. However, a study design in which these patterns were measured before the development of airflow limitation and the latter was assessed prospectively thereafter would require testing young children in whom induced sputum cannot be obtained and many years of follow-up. Also, the expression profiling studies and network analyses were performed on a mixed cell population from induced sputum, which is subject to sampling variability and contamination with saliva and squamous cells. Follow-up studies on highly purified cell populations should thus increase the sensitivity and precision/resolution of these analyses. Finally, sputum induction was not performed during severe exacerbations because it was deemed unsafe and unethical, and thus the mechanisms operating in severe exacerbations may be different from those reported herein.

These limitations notwithstanding, our findings for the first time provide a global perspective of the immunological networks that are operating during naturally occurring acute asthma exacerbations in children, and broadly characterize variations in these networks that are associated with deficits in baseline FEV1/FVC ratios. Our results identify a large range of logical candidates with well-characterized immunomodulatory properties (Table 2) for intervention studies to prevent the development of chronic airflow limitation in children with asthma.

\section{METHODS}

Study population and protocol design. The study population consisted of $218 \mathrm{mild} /$ moderate persistent asthmatic children aged 6-18 years who had at least one acute asthma exacerbation during the previous year. The study protocol included three visits. At enrollment, subjects had to be symptom-free; asthma severity and control was assessed by a study physician and adjustments were made, if necessary, to achieve national guideline recommendations for control. Baseline spirometry was assessed using an appropriately calibrated Jaeger spirometer (Erich Jaeger, Wurzburg, Germany). The ratio between FEV1 and FVC was used to assess airflow limitation at baseline, as suggested by 
Rasmussen et al. ${ }^{31}$ Skin test reactivity to local allergens was measured (Alternaria, dust mite mix (Dermatophagoides farinae plus D. pteronyssinus), olive, Bermuda, careless weed, mulberry, cockroach, cat, dog, mouse, and ragweed). After enrollment, the children were followed for 18 months or until their first asthma exacerbation, whichever came first.

A moderate exacerbation was defined with criteria equivalent to those recently proposed by the ATS/ERS consensus. ${ }^{44}$ If a child experienced symptoms of cough, dyspnea, chest tightness, and/or wheeze, they were instructed to initiate use of albuterol (two puffs, $90 \mathrm{mcg}$ per puff) by MDI every $20 \mathrm{~min}$ for up to $1 \mathrm{~h}$ and then every $4 \mathrm{~h}$ if necessary. For those who could use peak flow meter, a reading of $<80 \%$ of personal best was considered indicative of an exacerbation. If the subject could not get symptom relief after three treatments, parents were instructed to contact the study center immediately. These three conditions (symptoms of exacerbation, low peak flow readings, or lack of relief or persistence of symptoms after three treatments) were considered indicative of an exacerbation. Participants who met these criteria were scheduled for a visit at the study center within $24 \mathrm{~h}$. Ascertainment of an exacerbation was ultimately made by a study physician. This research was approved by the Institutional Review Board of the University of Arizona and informed consent was obtained for all participants.

Sputum induction. At the time of the acute exacerbation visit, a physical examination was performed by a study physician, lung function was assessed by spirometry, nasal secretions were collected to test for evidence of a picornavirus infection (online methods), and sputum was induced based on the techniques recommended by Gershman et al. ${ }^{45}$ with slight modification. After the measurement of spirometry pre- and post-bronchodilator, a peak flow measurement was made to determine the subject's baseline. Participants then inhaled 3\% saline for $1.5 \mathrm{~min}$, after which any accumulated saliva was discarded, followed immediately by coughing and expectoration of sputum. Peak flow was checked to assure that there had not been a $15 \%$ or greater drop after saline inhalation. This procedure was repeated at $2 \mathrm{~min}$ intervals six times. After the final inhalation of $3 \%$ saline and expectoration, a full spirometric maneuver was done in place of peak flow to assure no decline from baseline values. Only children whose FEV1 was $>70 \%$ of predicted were eligible for sputum induction. After 7-14 days of the acute episode, children were seen again in the study clinic and physical examination, nasal washes, and sputum collection were repeated as described above.

Sputum processing and RNA stabilization. Sputum was stored at $4{ }^{\circ} \mathrm{C}$ and processed within $1 \mathrm{~h}$ of collection. Briefly, whole sputum was diluted with a volume of $0.1 \%$ dithiothreitol (DTT-Sputolysin 10\%; Calbiochem, La Jolla, CA) equal to that of the weight of the original sample in grams, and incubated on a shaking water bath at $37^{\circ} \mathrm{C}$ for $15 \mathrm{~min}$ with intermittent aspiration by pipetting every $5 \mathrm{~min}$. Homogenized sputum was centrifuged $(800 \mathrm{~g})$ for $10 \mathrm{~min}$. The cell pellet was immediately resuspended in RNAlater Stabilization Reagent (Qiagen, Valencia, CA) as per manufacturer's recommendations. Total cell counts and differentials were determined from an aliquot of the original homogenized sample. Slides were prepared (Cytospin; Shandon, Runcorn, UK) and stained with a Wright-Giemsa stain. Differential cell counts were made by a blinded observer. Cells (100) were counted for each sample. Differential cell counts are expressed as percentages of total cells.

Microarray-based expression profiling studies. Total RNA from sputum samples stored in RNALater was extracted with TRIzol (Invitrogen, Carlsbad, CA) followed by RNeasy (Qiagen). In preliminary studies based on Bioanalyzer analysis, we noted some variation in the integrity of RNA from sputum. However, the microarray and real-time qRT-PCR protocols used in the study are based on random priming and are thus tolerant to variations in RNA quality. Total RNA samples $(n=20)$ were labeled and hybridized to Human Gene ST 1.0 microarrays (Affymetrix, Santa Clara, CA), at the Arizona Cancer Center Genomics Core, the University of Arizona. The microarray data are available from the Gene Expression Omnibus repository (accession number GSE19903).
The microarray data were preprocessed in Expression Consol software (Affymetrix) using the probe logarithmic intensity error algorithm with gc background subtraction, quantile normalization, and iterPLIER summarization. The preprocessed data were imported in the $\mathrm{R}$ language for statistical computing (http://www.r-project.org/), and variance stabilization was performed by adding the small constant 16 to all the data points, followed by $\log 2$ transformation.

Reverse engineering gene network analysis. The microarray data were filtered to select highly variable genes (top $10 \%$ on microarray3,247 genes) as well as the top 1,500 genes that differed in the respective responses (i.e., in subjects with $(n=10)$ or without $(n=10)$ deficits in enrollment FEV1/FVC ratios) according to their statistical ranking from a Bayesian $t$-test analysis. ${ }^{46}$ Network analysis was then performed on the filtered data set in all subjects using the weighted gene coexpression network analysis algorithm. ${ }^{22-24}$ The algorithm calculates absolute Pearson correlations for all pairwise gene-gene combinations across the test samples. The correlations are then raised to a power to emphasize stronger over weaker correlations. Genes that had a low overall correlation with the coexpression network were removed from the analysis (approximately $25 \%$ of initial genes removed). The topological overlap of the gene-gene correlations was calculated to quantify the extent that genes have similar overall patterns of correlations with other genes. The topological overlap similarity measure was subtracted from one to convert it into a distance measure and then analyzed by hierarchical clustering to group highly correlated genes into subnets (modules). The modules were defined from the dendrogram output of the cluster analysis using an automated algorithm (cutreeDynamic). The overall expression of the modules was compared in the respective responses using Gene Set Analysis without correcting for multiple testing. ${ }^{25}$ To determine if the modules were stable, we removed a randomly selected sample from the analysis just before the hierarchical clustering stage, and new modules were defined. This process was repeated an additional four times, and the stability of the modules was calculated as the proportion of genes from the original cluster that were detected in the same cluster, averaged over the five iterations.

Bioinformatics analysis of molecular signatures. The list of genes in the Th1-like/cytotoxic pathway (Supplementary Table S2 online) was interrogated for significant overlaps with the collection of 1,892 curated molecular signatures from the Molecular Signatures Database. The database contains annotated pathways from online databases, and molecular signatures from published microarray studies, thus captures a broad range of biological, cellular, and clinical states. Statistically significant overlaps were identified based on the Hypergeometric distribution. ${ }^{47}$ This analysis was performed online (http://www.broadinstitute.org/gsea/index.jsp).

qRT-PCR validation studies. Total RNA was reverse transcribed with a combination of random nonamers and oligo-dT priming using the Quantitect reverse transcription kit with integrated genomic DNA removal (Qiagen). qRT-PCR analysis was performed with Quantitect SyBr green (Qiagen) on the 7900 thermocycler (Applied Biosystems, Foster City, CA). The primer assay sequences for FCER1A were obtained from Primer Bank (http://pga.mgh.harvard.edu/primerbank/index. $\mathrm{html}$ ), and all other assays were obtained from Qiagen. Quantification was based on the relative standard curve method, and standards were prepared for each assay by serial diluting qRT-PCR products. The specificity of the qRT-PCR assays was confirmed by dissociation curve analysis and by testing negative RT control reactions. To select a housekeeping gene for normalization of the qRT-PCR data, we interrogated the microarray data for the following set of housekeeping genes: ACTB, ALAS1, B2M, EEF1A1, GAPDH, GUSB, HMBS, HPRT1, PGK1, PPIA, RPL13A, RPL27A, RPL37A, RPLP0, RRN18S, SDHA, TBP, TFRC, TUBB, YWHAZ. We selected $H M B S^{48}$ because it had the lowest variance. We also confirmed by qRT-PCR that the variance of HMBS expression was an order of magnitude lower than that of one of the most stably expressed genes in the genome (i.e., EEF1A1) ${ }^{49}$ and several orders of magnitude lower than RRN18S (18S rRNA). It is noteworthy that HMBS is expressed at 
low levels, ${ }^{48}$ thus providing more adequate adjustments for variations in RNA quality than highly expressed housekeeping genes.

Real-time qRT-PCR detection of iNKT cells and T cells in sputum. $\mathrm{T}$ cells and iNKT cells can be detected in sputum by qRT-PCR analysis of T-cell receptor transcripts. ${ }^{20}$ Most human iNKT cells express a V $\alpha 24-J \alpha 18$ (encoded by TRAV10 and TRAJ18; note J $\alpha 18$ was formally J $\alpha Q$ ) T-cell receptor alpha chain rearrangement paired with a V $\beta 11$ beta chain (encoded by TRBV25-1). ${ }^{50} \mathrm{~T}$ cells including iNKT cells express the TRBC2. ${ }^{20}$ The primer assay for detection of $\mathrm{V} \alpha 24$ was $\mathrm{V} \alpha 24$-F-AAGCATCTGACGACCTTCTTG, and V $\alpha 24$ R-AACAGGACCTCTCCCAGTATC. ${ }^{20}$ Primer assays for V $\beta 11$ (F, CCTCTGCTACGTGGGCTTT; R, GCCCATGGTTTGAGAACATT) and TRBC2 (F, AACCACTTCCGCTGTCAAGT; R, GCTGGTAAGACTCGGAGGTG) were designed with Primer3Plus (http://www.bioinformatics.nl/cgi-bin/primer3plus/primer3plus. cgi) to amplify ensemble transcript sequences (ENST00000390398, ENST00000390420), and concordance of ensemble sequences with published sequences from iNKT cells ${ }^{50}$ was confirmed using BLAST (http://blast.ncbi.nlm.nih.gov/Blast.cgi).

Statistical design and methodology. This study was designed with a two-stage analytical strategy. First, a case-control approach was used; two groups of 10 subjects each from both extreme tails of the FEV1/FVC distribution at enrollment were selected for the microarray profiling studies. In the second phase, qRT-PCR validation studies were performed on all available exacerbation sputum samples from the whole population $(n=40)$, and in these analyses, FEV1/FVC at enrollment, but also during the exacerbation and at convalescence, was treated as a quantitative trait.

Undetectable qRT-PCR data points were substituted with half the lowest value. Proportions were compared using Pearson $\chi^{2}$-test or Fisher's exact test. Association was assessed using nonparametric Spearman's rank-order correlation coefficient. Tobit regression was used to adjust for confounders when the dependent variable was left censored. Linear regression was used to adjust for controller medication use when the dependent variable was normally distributed. $t$-Tests, one-way analysis of variance were used to assess differences between groups for normally distributed data. Mann-Whitney $U$-test and Kruskal-Wallis $H$-test were used to assess differences between groups for non-normally distributed data. Wilcoxon's rank test was used for nonparametric paired data assessments. The data were analyzed using SPSS 17.0 (SPSS, Chicago, IL), STATA 10.0 (StataCorp, College Station, TX) and GraphPad Prism (GraphPad Software, La Jolla, CA).

SUPPLEMENTARY MATERIAL is linked to the online version of the paper at http://www.nature.com/mi

\section{ACKNOWLEDGMENTS}

We are grateful to Marilyn Halonen, Donata Vercelli, Michael Daines, and Yin Chen for critical reading of the paper, and to Jamie Goodwin and Monica Vasquez for support in obtaining the sputum samples. This work was supported by National Institutes of Health Grant HL080083.

\section{DISCLOSURE}

The authors declared no conflict of interest.

\section{(c) 2010 Society for Mucosal Immunology}

\section{REFERENCES}

1. Yunginger, J.W. et al. A community-based study of the epidemiology of asthma. Incidence rates, 1964-1983. Am. Rev. Respir. Dis. 146, 888-894 (1992).

2. Sears, M.R. et al. A longitudinal, population-based, cohort study of childhood asthma followed to adulthood. N. Engl. J. Med. 349, 1414-1422 (2003).

3. Strunk, R.C. et al. Mild to moderate asthma affects lung growth in children and adolescents. J. Allergy. Clin. Immunol. 118, 1040-1047 (2006).
4. Grol, M.H. et al. Risk factors for growth and decline of lung function in asthmatic individuals up to age 42 years. A 30-year follow-up study. Am. J. Respir. Crit. Care. Med. 160, 1830-1837 (1999).

5. Covar, R.A., Cool, C. \& Szefler, S.J. Progression of asthma in childhood. J. Allergy. Clin. Immunol. 115, 700-707 (2005).

6. Phelan, P.D., Robertson, C.F. \& Olinsky, A. The Melbourne Asthma Study: 1964-1999. J. Allergy. Clin. Immunol. 109, 189-194 (2002).

7. The Childhood Asthma Management Program Research Group. Long-term effects of budesonide or nedocromil in children with asthma. N. Engl. J. Med. 343, 1054-1063 (2000).

8. Guerra, S. et al. The course of persistent airflow limitation in subjects with and without asthma. Respir. Med. 102, 1473-1482 (2008).

9. James, A.L. et al. Decline in lung function in the Busselton Health Study: the effects of asthma and cigarette smoking. Am. J. Respir. Crit. Care. Med. 171, 109-114 (2005)

10. O'Byrne, P.M., Pedersen, S., Lamm, C.J., Tan, W.C. \& Busse, W.W. Severe exacerbations and decline in lung function in asthma. Am. J. Respir. Crit. Care. Med. 179, 19-24 (2009).

11. Bai, T.R., Vonk, J.M., Postma, D.S. \& Boezen, H.M. Severe exacerbations predict excess lung function decline in asthma. Eur. Respir. J. 30, 452-456 (2007).

12. Message, S.D. \& Johnston, S.L. Viruses in asthma. Br. Med. Bull. 61, 29-43 (2002).

13. Wark, P.A. et al. Asthmatic bronchial epithelial cells have a deficient innate immune response to infection with rhinovirus. J. Exp. Med. 201, 937-947 (2005).

14. Contoli, M. et al. Role of deficient type III interferon-lambda production in asthma exacerbations. Nat. Med. 12, 1023-1026 (2006).

15. Message, S.D. et al. Rhinovirus-induced lower respiratory illness is increased in asthma and related to virus load and Th1/2 cytokine and IL-10 production. Proc. Natl. Acad. Sci. USA 105, 13562-13567 (2008).

16. Brooks, G.D., Buchta, K.A., Swenson, C.A., Gern, J.E. \& Busse, W.W. Rhinovirus-induced interferon-gamma and airway responsiveness in asthma. Am. J. Respir. Crit. Care. Med. 168, 1091-1094 (2003).

17. Gern, J.E., Vrtis, R., Grindle, K.A., Swenson, C. \& Busse, W.W. Relationship of upper and lower airway cytokines to outcome of experimental rhinovirus infection. Am. J. Respir. Crit. Care. Med. 162, 2226-2231 (2000)

18. Kim, E.Y. et al. Persistent activation of an innate immune response translates respiratory viral infection into chronic lung disease. Nat. Med. 14, 633-640 (2008).

19. Akbari, O. et al. CD4+ invariant T-cell-receptor+ natural killer T cells in bronchial asthma. N. Engl. J. Med. 354, 1117-1129 (2006).

20. Vijayanand, P. et al. Invariant natural killer T cells in asthma and chronic obstructive pulmonary disease. N. Engl. J. Med. 356, 1410-1422 (2007).

21. Sears, M.R. Lung function decline in asthma. Eur. Respir. J. 30, 411-413 (2007).

22. Schadt, E.E. Molecular networks as sensors and drivers of common human diseases. Nature 461, 218-223 (2009).

23. Gargalovic, P.S. et al. Identification of inflammatory gene modules based on variations of human endothelial cell responses to oxidized lipids. Proc. Natl. Acad. Sci. USA 103, 12741-12746 (2006).

24. Bosco, A., McKenna, K.L., Firth, M.J., Sly, P.D. \& Holt, P.G. A network modeling approach to analysis of the Th2 memory responses underlying human atopic disease. J. Immunol. 182, 6011-6021 (2009).

25. Efron, B. \& Tibshirani, R. On testing the significance of sets of genes. Ann. Appl. Stat. 1, 107-109 (2007).

26. Schroder, K., Hertzog, P.J., Ravasi, T. \& Hume, D.A. Interferon-gamma: an overview of signals, mechanisms and functions. J. Leukoc. Biol. 75, 163-189 (2004).

27. Ross, A.J., Dailey, L.A., Brighton, L.E. \& Devlin, R.B. Transcriptional profiling of mucociliary differentiation in human airway epithelial cells. Am. J. Respir. Cell. Mol. Biol. 37, 169-185 (2007).

28. Hidalgo, L.G. et al. The transcriptome of human cytotoxic T cells: measuring the burden of CTL-associated transcripts in human kidney transplants. Am. J. Transplant 8, 637-646 (2008).

29. Bem, R.A. et al. Activation of the granzyme pathway in children with severe respiratory syncytial virus infection. Pediatr. Res. 63, 650-655 (2008).

30. Veillette, A., Dong, Z. \& Latour, S. Consequence of the SLAM-SAP signaling pathway in innate-like and conventional lymphocytes. Immunity 27, 698-710 (2007).

31. Rasmussen, F. et al. Risk factors for airway remodeling in asthma manifested by a low postbronchodilator FEV1/vital capacity ratio: a 
longitudinal population study from childhood to adulthood. Am. J. Respir. Crit. Care. Med. 165, 1480-1488 (2002).

32. Biron, C.A., Nguyen, K.B., Pien, G.C., Cousens, L.P. \& Salazar-Mather, T.P. Natural killer cells in antiviral defense: function and regulation by innate cytokines. Annu. Rev. Immunol. 17, 189-220 (1999).

33. Johnson, T.R., Hong, S., Van Kaer, L., Koezuka, Y. \& Graham, B.S. NKT cells contribute to expansion of CD8(+) T cells and amplification of antiviral immune responses to respiratory syncytial virus. J. Virol. 76, 4294-4303 (2002).

34. Cohen, N.R., Garg, S. \& Brenner, M.B. Antigen presentation by CD1 lipids, T cells, and NKT cells in microbial immunity. Adv. Immunol. 102, 1-94 (2009).

35. Kitamura, H. et al. The natural killer T (NKT) cell ligand alphagalactosylceramide demonstrates its immunopotentiating effect by inducing interleukin (IL)-12 production by dendritic cells and IL-12 receptor expression on NKT cells. J. Exp. Med. 189, 1121-1128 (1999).

36. Cui, J. et al. Requirement for Valpha14 NKT cells in IL-12-mediated rejection of tumors. Science 278, 1623-1626 (1997).

37. Coquet, J.M. et al. IL-21 is produced by NKT cells and modulates NKT cell activation and cytokine production. J. Immunol. 178, 2827-2834 (2007).

38. Parrish-Novak, J. et al. Interleukin 21 and its receptor are involved in NK cell expansion and regulation of lymphocyte function. Nature 408, 57-63 (2000).

39. Zhang, G. et al. Interleukin-10/interleukin-5 responses at birth predict risk for respiratory infections in children with atopic family history. Am. J. Respir. Crit. Care. Med. 179, 205-211 (2009).

40. Elsaesser, H., Sauer, K. \& Brooks, D.G. IL-21 is required to control chronic viral infection. Science 324, 1569-1572 (2009).
41. Akbari, O. et al. Essential role of NKT cells producing IL-4 and IL-13 in the development of allergen-induced airway hyperreactivity. Nat. Med. 9, 582-588 (2003).

42. Steinman, R.M. \& Mellman, I. Immunotherapy: bewitched, bothered, and bewildered no more. Science 305, 197-200 (2004).

43. Davis, M.M. A prescription for human immunology. Immunity 29, 835-838 (2008).

44. Reddel, H.K. et al. An official American Thoracic Society/European Respiratory Society statement: asthma control and exacerbations: standardizing endpoints for clinical asthma trials and clinical practice. Am. J. Respir. Crit. Care. Med. 180, 59-99 (2009).

45. Gershman, N.H., Wong, H.H., Liu, J.T., Mahlmeister, M.J. \& Fahy, J.V. Comparison of two methods of collecting induced sputum in asthmatic subjects. Eur. Respir. J. 9, 2448-2453 (1996).

46. Smyth, G.K. Linear models and empirical Bayes methods for assessing differential expression in microarray experiments. Stat. Appl. Genet. Mol. Biol. 3: Article 3 (2004).

47. Huang da, W., Sherman, B.T. \& Lempicki, R.A. Bioinformatics enrichment tools: paths toward the comprehensive functional analysis of large gene lists. Nucleic Acids Res. 37, 1-13 (2009).

48. Vandesompele, J. et al. Accurate normalization of real-time quantitative RT-PCR data by geometric averaging of multiple internal control genes. Genome Biol. 3, RESEARCH0034 (2002).

49. Warrington, J.A., Nair, A., Mahadevappa, M. \& Tsyganskaya, M. Comparison of human adult and fetal expression and identification of 535 housekeeping/maintenance genes. Physiol. Genomics 2, 143-147 (2000).

50. Brigl, M. et al. Conserved and heterogeneous lipid antigen specificities of CD1d-restricted NKT cell receptors. J. Immunol. 176, 3625-3634 (2006). 